\title{
Electronic health records and adverse drug events after patient transfer
}

\author{
K S Boockvar, ${ }^{1,2,3}$ E E Livote, ${ }^{1}$ N Goldstein, ${ }^{1,2}$ J R Nebeker, ${ }^{4}$ A Siu, ${ }^{1,2}$ T Fried $^{5,6}$
}

${ }^{1}$ Geriatric Research, Education, and Clinical Center, James J Peters Veterans Affairs Medical Center, Bronx, New York, USA ${ }^{2}$ Departments of Geriatrics and Palliative Medicine, Mount Sinai School of Medicine, New York, New York, USA

${ }^{3}$ The Jewish Home Lifecare, New York, New York, USA ${ }^{4}$ VA Geriatrics Research, Education, and Clinical Center, Salt Lake City, Utah, USA ${ }^{5}$ Yale University School of Medicine, New Haven,

Connecticut, USA ${ }^{6}$ Clinical Epidemiology Research Center, VA Connecticut Healthcare System, West Haven, Connecticut, USA

\section{Correspondence to}

Kenneth S Boockvar, James J Peters VA Medical Center, 130 West Kingsbridge Road, Bronx, NY 10468, USA;

kenneth.boockvar@mssm.edu.

Accepted 31 August 2009 Published Online First 19 August 2010

\section{ABSTRACT}

Background Our objective was to examine the frequencies of medication error and adverse drug events (ADEs) at the time of patient transfer in a system with an electronic health record (EHR) as compared with a system without an EHR. It was hypothesised that the frequencies of these events would be lower in the EHR system because of better information exchange across sites of care.

Methods 469 patients transferred between seven nursing homes and three hospitals in New York and Connecticut between 1999 and 2005 were followed retrospectively. Two groups of patients were compared: US Veterans Affairs (VA) patients, with an EHR, and nonVA patients, without an EHR, on the following measures: (1) medication prescribing discrepancies at nursing home/hospital transfer, (2) high-risk medication discrepancies and (3) ADEs caused by medication discrepancies according to structured medical record review by pairs of physician and pharmacist raters.

Results The overall incidence of ADE caused by medication discrepancies was 0.20 per hospitalisation episode. After controlling for demographic and clinical covariates, there were no significant differences between VA and non-VA groups in medication discrepancies (mean difference $0.02 ; 95 \% \mathrm{Cl}-0.81$ to 0.85 ), high-risk medication discrepancies $(-0.18 ; 95 \% \mathrm{Cl}-0.22$ to 0.58$)$ or occurrence of an ADE caused by a medication discrepancy (OR 0.96; $95 \% \mathrm{Cl} 0.18$ to 5.01 ).

Conclusions There was no difference, with and without an EHR, in the occurrence of medication discrepancies or ADEs caused by medication discrepancies at the time of transfer between sites of care. Reducing such problems may require specialised computer tools to facilitate medication review.

\section{INTRODUCTION}

Patient transfers or handoffs between sites of care are common in healthcare systems and are associated with communication lapses that cause adverse events. Discrepancies in medication prescribing are common lapses that occur during transfer and such discrepancies cause adverse drug events (ADEs). ${ }^{1-3}$ Standard medication review and reconciliation procedures have been tested to reduce such $\mathrm{ADEs}$ in several countries. ${ }^{4}{ }^{5}$ In the US, the Joint Commission established medication reconciliation during patient handoffs as a safety standard in $2006{ }^{6}$

An electronic health record (EHR) portable or accessible across sites of care should improve communication between sites of care and reduce errors by enabling providers to see more complete prescribing information, read past and current notes, and prescribe medication electronically. ${ }^{7}$ According to the US Institute of Medicine, exchange of patient care data and communication among care settings are basic and required functions of an EHR. ${ }^{8}$ As a prominent example, the US Department of Veterans Affairs (VA) has an EHR that enables VA providers to view healthcare encounter notes, summaries, orders, pharmacy, radiology and laboratory results from any VA setting. Communication forms to exchange health information between sites of care are unnecessary. Yet, whether an EHR like the VA's changes the likelihood of medication discrepancies or ADEs from medication discrepancies at the time of patient handoffs is unknown.

The objective of this study was to examine the frequencies of medication discrepancies and ADEs from medication discrepancies at the time of patient handoff within the VA system as compared with outside the VA system, where paper forms, sometimes handwritten, are used to communicate health information. We hypothesised that the frequencies of these events would be lower in the VA system owing to its advanced EHR, as compared with our non-VA study sites where an EHR was not available for information exchange.

\section{METHODS}

\section{Setting and participants}

The study was an observational cohort study in which two groups of patients were compared: VA, with an EHR, and non-VA, without an EHR, in New York and Connecticut between 1999 and 2005. The VA group consisted of patients from four VA nursing homes who were admitted to two VA hospitals that were the primary referral hospitals for the nursing homes. The non-VA group consisted of patients from three non-VA nursing homes who were admitted to one non-VA hospital that was the primary referral hospital for the nursing homes. All VA and non-VA study hospitals were academic centres located in urban areas, and all study nursing homes were located in urban or suburban areas.

Eligible patients were individuals transferred from nursing home to hospital and admitted, and who remained in the hospital for at least $24 \mathrm{~h}$. Individuals were included whether or not they survived to hospital discharge and whether or not they returned to the nursing home from which they originated. Institutional review boards of Mount Sinai School of Medicine, James J Peters VA Medical Center, VA Connecticut Healthcare System and Jewish Home Lifecare approved a waiver of informed consent, since data were collected by retrospective medical record review. 


\section{Measurements}

\section{Medication discrepancies}

Research personnel reviewed nursing home and hospital records to identify medication prescribing discrepancies at transitions between sites of care, which were hospital-to-nursing home transfer and nursing home-to-hospital transfer if the patient survived to discharge and returned to the same nursing home. Records reviewed included medication orders, transfer documents, medication administration information and discharge instructions. A discontinuation, dose change, frequency change, route change or substitution for a medication with the same indication at the time of transition was labelled a prescribing discrepancy. We included substitutions caused by nursing home and hospital drug formulary differences, but excluded substitutions between generic and brand-name versions of the same drug. Discrepancies in 14 drug classes were classified as high risk (angiotensin blockers, antiarrhythmic agents, anticoagulants, antiepileptic agents, antiprostate agents, antipsychotics, calcium blockers, insulin, metronidazole, nitrates, non-opioid analgesics, opioid analgesics, sedative/hypnotics and thyroid replacements). These were compiled from the Institute for Healthcare Improvement's High Alert medication drug classes, ${ }^{9}$ high-risk drug classes for nursing home patients ${ }^{10} 11$ and other classes with higher likelihood of causing a discrepancy-related $\mathrm{ADE} .^{12}$ Topical agents, vitamins, minerals and as-needed medications not in a high-risk drug class were excluded. Laxatives were excluded because of ascertainment differences between VA and non-VA groups.

\section{Adverse drug events caused by medication discrepancies}

A subsample of nursing home and hospital records was reviewed for up to 2 months after each transition between the care sites for ADEs caused by medication discrepancies. The subsample was randomly selected, but patients from nursing homes with fewer hospitalisations were oversampled. Two trained clinician investigators (two physicians or one physician and one pharmacist) reviewed records for medical incidents that were defined in advance, including new or worse bleeding, congestive heart failure, delirium, diarrhoea, dyspnoea, fall, decrease in alertness, incontinence, pain, rash, urinary retention, vomiting, blood pressure abnormalities (new systolic blood pressure $>185$ or $<95$, diastolic blood pressure $>105$ ), fever (temperature $>100.5 \mathrm{~F}$ ), and abnormal tests of kidney function (creatinine increase $>0.5$ ), liver function (doubling of aspartate aminotransferase or alanine aminotransferase) or over-anticoagulation (international normalised ratio >4.0). Other laboratory abnormalities (eg, hypoglycaemia or hyperglycaemia, hyperkalaemia) were recorded if symptomatic or if they caused a cardiac arrhythmia.

Each rater recorded whether a medical incident could have been caused by a prescribing discrepancy at the time of transfer between care sites using structured implicit review. Implicit review criteria included (1) whether the incident was a physiologically possible consequence of the medication prescribing discrepancy, (2) whether there was a note in the medical record that suggested that a medication discrepancy caused the incident, (3) the time interval between incident and discrepancy, (4) whether the incident could have been caused by something other than a medication discrepancy and (5) whether the patient's condition improved after correction of the medication discrepancy.

The two raters discussed each event and provided a consensus rating using a six-point Likert scale, with one indicating "little or no" certainty and six indicating "almost total" certainty ${ }^{13}$ that a drug discrepancy caused an ADE. For this study, ADEs were defined as events for which the consensus rating was $\geq 4$. Raters then decided whether an $\mathrm{ADE}$ was the result of a prescribing error according to whether there was an appropriate clinical rationale for the prescribing discrepancy or the discrepancy deviated from prescribing norms, as determined by discussion consensus. Finally, raters scored ADE severity, using modified National Coordinating Council for Medication Error Reporting and Prevention categories, indicating whether the $\mathrm{ADE}$ caused symptoms, a prolonged or additional hospital stay, permanent harm or death.

\section{Patient characteristics}

Information was collected on patient age, gender, race and number of prescribed medications from the nursing home record. A score for burden of chronic disease, adapted from Charlson et al, ${ }^{14}$ was calculated from chronic medical problems listed in the nursing home record. Information on hospitalisation episode diagnoses, time of admission (8:00-18:00 Monday-Friday vs off-hours) and hospital length of stay were obtained from the hospital record. An illness severity score, modified from the Acute Physiology and Chronic Health Evaluation score, ${ }^{15}$ was calculated from initial laboratory data and vital signs in the hospital record.

\section{Analysis}

Because a single patient could contribute more than one hospitalisation episode to the study, the unit of analysis was hospitalisation episode. Baseline characteristics of VA and non-VA patients and characteristics of their hospitalisation episodes were compared, using the $t$ test for continuous variables and $\chi^{2}$ test for categorical variables. The main study outcomes were medication discrepancies, high-risk medication discrepancies and ADEs caused by medication discrepancies during transfer to or from the hospital. We calculated VA/non-VA group differences in the mean number of medication discrepancies and mean number of highrisk medication discrepancies, and the VA/non-VA group odds that an $\mathrm{ADE}$ caused by a medication discrepancy occurred during transfer, each with 95\% CIs. To adjust for differences between VA and non-VA patients, regression models were fitted in which VA or non-VA group was the key independent variable; gender, age, prehospitalisation number of medications, Charlson comorbidity score, Acute Physiology and Chronic Health Evaluation score, offhours admission, admission diagnoses and duration of follow-up were covariates; and medication discrepancies, high-risk discrepancies and $\mathrm{ADE}$ were the dependent variables. We used generalised estimating equations to account for clustering of observations within patients and facilities. 95\% CIs, $\mathrm{p}$ values, odds ratios and

Table 1 Characteristics of hospitalised nursing home patients and their hospitalisations, stratified by VA/non-VA group

\begin{tabular}{lll}
\hline & VA & Non-VA \\
\hline Patients (n) & 226 & 243 \\
Age (mean years (SD)) & $70.0(13.0)$ & $82.8(10.6)^{*}$ \\
Male (\%) & $97.3 \%$ & $25.4 \% \%^{*}$ \\
Hospitalisations (N) & 331 & 387 \\
Hospitalisations/patient (N/n) & 1.5 & 1.6 \\
Prehospitalisation medications & $6.0(3.1)$ & $6.2(2.9)$ \\
(mean number (SD)) & & \\
Hospital admission off-hours§ (\%) & $63.4 \%$ & $52.3 \% \dagger$ \\
$\begin{array}{l}\text { Hospital length of stay (median days } \\
\text { (range)) }\end{array}$ & $7(1-296)$ & $6(1-98) \neq$ \\
\hline
\end{tabular}

${ }^{*} p<0.001$ for comparison with VA group.

$+p<0.01$ for comparison with VA group.

$\neq p \leq 0.05$ for comparison with VA group.

§Not during regular business hours (8:00-18:00 Monday-Friday).

VA, US Department of Veterans Affairs. 
Table 2 Medication discrepancies and ADEs caused by medication discrepancies in hospitalised nursing home patients, stratified by VA/non-VA group

\begin{tabular}{|c|c|c|c|c|}
\hline & VA & Non-VA & Unadjusted difference & Adjusted difference* \\
\hline Medication discrepancies† (mean (SD)) & $2.62(2.16)$ & $2.55(2.18)$ & Diff $0.07(-0.26$ to 0.42$)$ & Diff $0.02(-0.81$ to 0.85$)$ \\
\hline High-risk medication discrepancies† (mean (SD)) & $0.96(1.07)$ & $0.75(0.92)$ & Diff $0.21(0.05$ to 0.38$) \S$ & Diff $-0.18(-0.22$ to 0.58$)$ \\
\hline ADE caused by a medication discrepancy $\ddagger$ ( $\%$ of & $18.1 \%$ & $21.5 \%$ & OR 0.83 (0.44 to 1.59$)$ & OR 0.96 (0.18 to 5.01$)$ \\
\hline
\end{tabular}

hospitalisation episodes)

(Subsample $\mathrm{n}=127$ VA; $\mathrm{n}=177$ non-VA)

*All models were adjusted for gender, age, prehospitalisation number of medications, Charlson comorbidity score, APACHE score, off-hours admission, admission diagnoses and duration of follow-up.

†At the time of hospital admission.

$\ddagger$ At the time of hospital admission or discharge.

$\S p=0.01$ for $V A /$ non-VA group comparison.

$A D E$, adverse drug events; VA, US Department of Veterans Affairs.

the c statistic were calculated using standard formulae. All analyses were performed using SAS software version 9.1.

\section{RESULTS}

The study sample consisted of 226 VA nursing home patients hospitalised 331 times and 243 non-VA nursing home patients hospitalised 387 times. Characteristics of patients and their hospital stays are shown in table 1. VA patients were younger, more likely to be men and more likely to be admitted during offhours. The most common reasons for hospitalisation in both groups were pneumonia (in 18\% of cases), urinary tract infection $(17 \%)$, dehydration $(11 \%)$, exacerbations of congestive heart failure (8.5\%) and chronic obstructive pulmonary disease (4.0\%). There was no difference in prehospitalisation number of medications prescribed. Median hospital length of stay was longer by 1 day in the VA group than in the non-VA group.

The total number of prescribing discrepancies observed in both study groups was 1854 at the time of nursing home-tohospital transfer and 1059 at the time of hospital-to-nursing home transfer, for a mean (SD) of 2.58 (2.17) and 1.61 (1.85) discrepancies per transfer, respectively. Sixty-four per cent of discrepancies were drug discontinuations, 19\% were dosage or frequency changes, $12 \%$ were substitutions for a medication with the same indication and 5\% were other types. In the subsample of records reviewed for ADEs, the total number of ADEs caused by discrepancies at the time of nursing home-tohospital or hospital-to-nursing home transfer was 61, for an $\mathrm{ADE}$ incidence of 0.20 (95\% CI 0.16 to 0.25 ) per hospitalisation episode. Fifty-two per cent of prescribing discrepancies that caused $\mathrm{ADEs}$ were considered to be appropriate prescribing changes and $48 \%$ were considered to be prescribing errors. Errors included wrong omissions (46\%), errors in drug frequency $(46 \%)$ and errors in drug dosage (8\%). Forty-six per cent of ADEs were asymptomatic, $52 \%$ were associated with symptoms and $3 \%$ caused a prolonged or an additional hospital stay. No ADE resulted in permanent disability or death.

In unadjusted comparisons, the VA group, as compared with the non-VA group, had a similar number of transfer-related medication discrepancies (mean 2.62 vs 2.55; difference 0.07 ( $95 \% \mathrm{CI}-0.26$ to 0.42$) ; \mathrm{p}=0.66)$ and a higher number of highrisk discrepancies (mean 0.96 vs 0.75 ; difference 0.21 (95\% CI 0.05 to 0.38$) ; \mathrm{p}=0.01$ ) per hospitalisation episode; and had a similar percentage of hospitalisation episodes in which an $\mathrm{ADE}$ caused by a medication discrepancy occurred (18.1\% vs $21.5 \%$; OR 0.83 (95\% CI 0.44 to 1.59); $\mathrm{p}=0.57$ ) (table 2). In adjusted comparisons, after controlling for patient demographic and clinical covariates, there were no significant differences between VA and non-VA groups in any outcome (medication discrepancies, high-risk medication discrepancies or ADEs caused by medication discrepancies) (table 2). The $\mathrm{c}$ statistic for our ADE outcome model was 0.682 , which indicates fair discrimination. In addition, there were no significant differences between VA and non-VA groups in $\mathrm{ADE}$ severity or in $\mathrm{ADEs}$ caused by prescribing errors (table 3 ).

Examples of ADEs caused by three common types of medication discrepancies are shown in table 3. The first type of discrepancy was a purposeful prescribing change with an appropriate clinical rationale that ultimately caused harm-for example, a case of an omission of transdermal nitroglycerin in a VA patient with low blood pressure at the time of hospital admission that caused harm when the patient's angina relapsed. The second discrepancy type was a purposeful prescribing change that lacked an appropriate clinical rationale-for example, a case of omission of transdermal fentanyl in a VA patient with chronic pain that caused pain relapse. The third discrepancy type was an inadvertent transcribing error associated with a provider incorrectly re-entering a medication order at the time of transfer-for example, a case of dosage increase in phenytoin in a VA patient that caused a supratherapeutic phenytoin level. As shown, there were ADEs caused by each of these types of discrepancies in the VA group that were not averted by the VA's EHR.

\section{DISCUSSION}

Substantial worldwide resources are being directed towards building capacity for electronic health information exchange.

Table 3 Complications of ADEs caused by medication discrepancies, stratified by VA/non-VA group

\begin{tabular}{|c|c|c|c|}
\hline ADE caused: & VA & Non-VA* & Example: discrepancy (ADE) \\
\hline \multirow[t]{2}{*}{ No symptoms $(\%)$} & 42 & 48 & VA: phenytoin dosage increase (supratherapeutic phenytoin level) \\
\hline & & & Non-VA: clonidine omitted (hypertension) \\
\hline Symptoms only $(\%)$ & 54 & 50 & VA: fentanyl transdermal omitted (pain) \\
\hline \multirow[t]{2}{*}{ Prolonged or additional hospital stay (\%) } & 4 & 2 & VA: nitroglycerin transdermal omitted (cardiac ischaemia, hospital readmission) \\
\hline & & & Non-VA: colchicine omitted (gout flare, prolonged hospital stay) \\
\hline Permanent harm or death (\%) & 0 & 0 & None \\
\hline
\end{tabular}

${ }^{*} p \geq 0.05$ for all comparisons with VA group, by $\chi^{2}$ test.

$A D E$, adverse drug events; VA, US Department of Veterans Affairs. 
These efforts complement longstanding electronic health information exchange capacity within the US VA system. In the USA, the existence of the VA system with its mature EHR alongside non-VA settings without an EHR afford an opportunity to test whether the EHR is associated with better prescribing at the time of interfacility patient transfer. Our hypothesis was refuted by the finding that hospitalised nursing home residents in the VA setting had no difference in the occurrence of medication discrepancies, high-risk medication discrepancies or $\mathrm{ADEs}$ caused by medication discrepancies at the time of transfer as compared with those in the non-VA setting.

No previous studies have isolated the effect of an EHR on prescribing outcomes at the time of transfer between sites of care. Our findings are consistent with a previous study that demonstrated that $\mathrm{ADEs}$ from errors in medication ordering are common even in a highly computerised system. ${ }^{16}$ Barriers to effective medication reconciliation and review at the time of transfer in a computerised setting include computerised medication information that is incomplete or unclear, a reliance on the computer by providers that leads to less thorough patient interviews and less careful medication reviews, poor computer interface design and competing provider tasks such as high volume or very ill patients. At the time of this study, the VA EHR provided excellent access to many types of information but did not provide tools that facilitated medication review or reconciliation. Yet, our study and others ${ }^{17-20}$ suggest that reducing medication discrepancies at the time of intersite transfer has the potential to prevent ADEs. Procedures that might improve this process in computerised and non-computerised settings include dedicating staff to complete standardised medication reconciliation and review, integrating the task into other provider tasks such as documentation or ordering, and alerting providers in real time when a prescribing discrepancy is detected that has a higher likelihood of causing an $\mathrm{ADE}$.

A limitation of this study is the small overall number of ADEs. However, the CIs for the differences in medication discrepancies and high-risk medication discrepancies were small, suggesting that the null finding is robust. In addition, the small fraction of severe ADEs in this study is concordant with reviews that indicate that serious $\mathrm{ADEs}$ make up only a small fraction of total ADEs. ${ }^{21} 22$ It is possible that our no-difference finding could have occurred because of a lower than expected number of medication discrepancies in the non-VA group, which had better information exchange than that observed in older studies. ${ }^{23}$ Our null finding could also have been influenced by ascertainment bias, whereby the VA's comprehensive and readable EHR increased the likelihood of finding ADEs in the narrative record. However, this should not have affected our ascertainment of medication discrepancies or of high-risk medication discrepancies, which did not involve review of the narrative record. It should be noted that data from this study preceded the US Joint Commission 2006 medication reconciliation standard, when most facilities implemented a procedure for medication reconciliation. "Nevertheless, because organizations have had difficulty implementing the process, the Joint Commission stopped citing medication reconciliation deficiencies in its accreditation surveys and is currently revising the standard." ${ }^{24}$ Finally, our no difference finding could have been specific to the small sample of facilities studied and not representative.

In conclusion, implementation of an EHR, and ensuring interoperability among EHRs, would not alone be expected to resolve the problem of $\mathrm{ADEs}$ from medication discrepancies at the time of intersite transfer. Reducing such problems may require specialised computer tools to facilitate medication review and reconciliation, and attention to EHR usability and implementation to reduce provider and system barriers to accurate medication prescribing.

Acknowledgements The authors would like to acknowledge the assistance of Toy Mordiglia and Bella Fridman in data collection.

Funding Financial support was provided by the VA Health Services Research and Development Service, the New York State Department of Health and the Mount Sinai Practice Improvement Cluster. Dr. Goldstein was supported by 5K23AG025933-04 from NIH.

Competing interests The authors have no competing interests to report. The sponsors had no role in the design or conduct of the study; collection, management, analysis or interpretation of the data; or in the preparation, review or approval of the manuscript. Dr Boockvar had full access to study data and takes responsibility for the integrity of the data and the accuracy of the data analysis.

Ethics approval This study was conducted with the approval of the Mount Sinai School of Medicine, James J Peters VA Medical Center, VA Connecticut Healthcare System and Jewish Home Lifecare.

Contributors KSB: conception and design, analysis and interpretation of data; drafting the article and revising it critically for important intellectual content; and final approval of the version to be published. KSB had full access to study data and takes responsibility for the integrity of the data and the accuracy of the data analysis (guarantor). EEL: analysis and interpretation of data, revising the article critically for important intellectual content and final approval of the version to be published. JRN analysis and interpretation of data, revising the article critically for important intellectual content and final approval of the version to be published. NG: analysis and interpretation of data, revising the article critically for important intellectual content and final approval of the version to be published. AS: analysis and interpretation of data, revising the article critically for important intellectual content and final approval of the version to be published. TF: analysis and interpretation of data, revising the article critically for important intellectual content and final approval of the version to be published. Toy Mordiglia (non-author): data collection. Bella Fridman (non-author): data collection.

Provenance and peer review Not commissioned; externally peer reviewed.

\section{REFERENCES}

1. Glintborg B, Andersen SE, Dalhoff K. Insufficient communication about medication use at the interface between hospital and primary care. Qual Saf Health Care 2007;16:34-9.

2. Boockvar K, Fishman E, Kyriacou CK, et al. Adverse events due to discontinuation in drug use and dose changes in patients transferred between acute and long-term care facilities. Arch Intern Med 2004;164:545-50.

3. Coleman EA, Smith JD, Raha D, et al. Posthospital medication discrepancies: prevalence and contributing factors. Arch Intern Med 2005;165:1842-7.

4. Danish Society for Patient Safety: Operation Life Denmark. http://patientsikkerhed. $\mathrm{dk} / \mathrm{en} /$ about_the_danish_society_for_patient_safety/activities/ (accessed 1 July 2010).

5. Vira T, Colquhoun M, Etchells E. Reconcilable differences: correcting medicationerrors at hospital admission and discharge. Qual Saf Health Care 2006;15:122-6.

6. Joint Commission Hospital National Patient Safety Goal \#8. http://www. jointcommission.org/AccreditationPrograms/Hospitals/NPSG/ laccessed 30 June 2010).

7. Burton LC, Anderson GF, Kues IW. Using electronic health records to help coordinate care. Milbank 0 2004:82:457-81, table of contents.

8. Tang PC. Key capabilities of an electronic health record system. Washington, DC: National Academy Press: Institute of Medicine. Committee on Data Standards for Patients Safety, 2003

9. Federico F. Preventing harm from high-alert medications. Jt Comm J Qual Patient Saf 2007:33:537-42.

10. Cooper JW. Adverse drug reaction-related hospitalizations of nursing facility patients: a 4-year study. South Med J 1999:92:485-90.

11. Gurwitz JH, Field TS, Avorn J, et al. Incidence and preventability of adverse drug events in nursing homes. Am J Med 2000;109:87-94.

12. Boockvar K, Liu S, Goldstein N, et al. Prescribing discrepancies likely to cause adverse drug events after patient transfer. Qual Saf Health Care 2009:18:32-6.

13. Brennan TA, Localio AR, Leape LL, et al. Identification of adverse events occurring during hospitalization. A cross-sectional study of litigation, quality assurance, and medical records at two teaching hospitals. Ann Intern Med 1990;112:221x-6.

14. Charlson ME, Pompei P, Ales KL, et al. A new method of classifying prognostic comorbidity in longitudinal studies: development and validation. J Chronic Dis 1987:40:373-83

15. Knaus WA, Draper EA, Wagner DP, et al. APACHE II: a severity of disease classification system. Crit Care Med 1985:13:818-29.

16. Nebeker JR, Hoffman JM, Weir CR, et al. High rates of adverse drug events in a highly computerized hospital. Arch Intern Med 2005;165:1111-16.

17. Whittington J, Cohen H. OSF healthcare's journey in patient safety. Qual Manag Health Care 2004;13:53-9. 
18. Kramer JS, Hopkins PJ, Rosendale JC, et al. Implementation of an electronic system for medication reconciliation. Am J Health Syst Pharm 2007;64:404-22.

19. Boockvar KS, Carlson LaCorte H, Giambanco V, et al. Medication reconciliation for reducing drug-discrepancy adverse events. Am J Geriatr Pharmacother 2006; $4: 236-43$.

20. Bartick M, Baron D. Medication reconciliation at Cambridge Health Alliance: experiences of a 3-campus health system in Massachusetts. Am J Med Qual 2006;21:304-6.

21. Layde PM, Maas LA, Teret SP, et al. Patient safety efforts should focus on medical injuries. JAMA 2002:287:1993-7.
22. Leape LL, Berwick DM, Bates DW. What practices will most improve safety? Evidence-based medicine meets patient safety. JAMA 2002;288:501-7.

23. Jones JS, Dwyer PR, White LJ, et al. Patient transfer from nursing home to emergency department: outcomes and policy implications. Acad Emerg Med 1997:4:908-15.

24. Medication reconciliation. National Patient Safety Goal field review results. Joint Commission Online June 2, 2010. Available at http://www.jointcommission.org/NR/ rdonlyres/17295FE3-6643-48E6-89A5-C629609E3F36/0/jconlineJune210.pdf (accessed June 30, 2010). 